Cahiers $d u$ MONDE RUSSE

\section{Cahiers du monde russe}

Russie - Empire russe - Union soviétique et États indépendants

$43 / 4 \mid 2002$

Intellectuels et intelligentsia

\title{
Antonella Salomoni, Il pane quotidiano
}

\section{Alessandro Stanziani}

\section{OpenEdition \\ Journals}

Édition électronique

URL : https://journals.openedition.org/monderusse/4021

DOI : $10.4000 /$ monderusse. 4021

ISSN : $1777-5388$

\section{Éditeur}

Éditions de l'EHESS

\section{Édition imprimée}

Date de publication : 30 décembre 2002

Pagination : 708-710

ISBN : 2-7132-1796-2

ISSN : $1252-6576$

\section{Référence électronique}

Alessandro Stanziani, «Antonella Salomoni, II pane quotidiano », Cahiers du monde russe [En ligne], 43/4 | 2002, mis en ligne le 17 juin 2009, consulté le 03 septembre 2022. URL : http://

journals.openedition.org/monderusse/4021 ; DOI : https://doi.org/10.4000/monderusse.4021

Ce document a été généré automatiquement le 3 septembre 2022

Tous droits réservés 


\title{
Antonella Salomoni, Il pane quotidiano
}

\author{
Alessandro Stanziani
}

\section{RÉFÉRENCE}

Antonella SALOMONI, Il pane quotidiano. Ideologia e congiuntura nella Russia sovietica (1917-1921). Bologne, Il Mulino, 2001, 324 p.

1 Antonella Salomoni nous a habitués à des études qui ont le mérite, rare, de conjuguer érudition, rigueur historique et capacité novatrice. C'est le cas du Lenin censuré (« Lenin censuré : deux fragments inédits de décembre 1917 », Cahiers du Monde russe et soviétique, 1, 1986, p. 71-94), des archives totalitaires (« Un savoir historique d'État: les archives soviétiques ", Annales HSS, 1, 1995, p. 3-27) ou encore du paysan Bondarev (Il lavoro del pensiero. Il contadino Timofej Bondarev e lo scrittore Lev Tolstoj, Name, 2001) et du mouvement tolstoïen en Italie (Il pensiero religioso e politico di Tolstoj in Italia, 1886-1910, Olschki, 1996). Cette fois-ci, il s'agit d'une étude sur le communisme de guerre. Cette phase initiale du pouvoir bolchevik en Russie a donné vie, à partir des années 1920 en URSS, puis, jusqu'à nos jours en URSS comme en « Occident ", à une âpre controverse politique, idéologique et historiographique à la fois. La question est de savoir si cet ensemble de mesures connues comme "communisme de guerre»a été dicté par l'émergence de la guerre civile ou s'il découle de l'idéologie bolchevique. Selon la réponse apportée, toute l'interprétation de la révolution d'Octobre, des premières années du régime soviétique, de Lenin et de son rapport à Stalin - en un mot, la signification elle-même de l'expérience soviétique - varie. Si en effet on suit la première interprétation, alors seules les nécessités imposées par la guerre expliquent les mesures extrêmes adoptées entre 1918 et 1921. Une fois la guerre civile terminée, Lenin opte pour une attitude modérée et introduit la NEP. Au contraire, la deuxième interprétation estime que l'idéologie marxiste, dans sa variante bolchevique, pousse les dirigeants 
communistes à adopter des mesures qui visent à instaurer le régime souhaité. Rien à voir donc avec les contraintes de la guerre.

2 Antonella Salomoni dépasse d'un bond des décennies de querelles en nous montrant de prime abord que le "communisme de guerre» constitue une véritable invention historiographique. Lenin, puis d'autres dirigeants bolcheviks, n'en parlent qu'à partir de 1921, de manière à légitimer la NEP et à l'opposer aux politiques précédentes. L'enjeu de cette «invention» est de taille: il ne s'agit rien de moins que de définir la doctrine communiste, le régime soviétique et finalement d'encadrer la révolution d'Octobre dans un schéma historico-idéologique conforme aux objectifs politiques courants.

3 Afin de démontrer cette thèse, le premier chapitre de l'ouvrage retrace l'historique des débats sur le communisme de guerre des années 1920 jusqu'à nos jours, en passant par les « révisions» staliniennes, puis celles des années 1960 ou encore de l'époque de la " perestroïka».

4 Le deuxième chapitre évoque les théories des principaux dirigeants soviétiques (Larin, Lenin, Groman) au sujet du Plan, du capitalisme d'État et de l'économie de guerre. L'auteur montre l'influence majeure exercée par l'expérience allemande au cours de la Première Guerre mondiale. De ce point de vue, et c'est là une autre nouveauté majeure de cet ouvrage, le communisme de guerre ne saurait véritablement se comprendre qu'en étant inclus dans la dynamique plus vaste de l'économie et de la politique européennes.

5 Confirmation de cette hypothèse, le troisième et le quatrième chapitres sont respectivement consacrés aux théories et aux pratiques de «naturalisation» de l'économie au cours de la guerre civile. Processus en partie inscrit dans l'inflation consécutive à la guerre, mais qui reçoit en Russie un accueil tout à fait particulier chez des responsables qui trouvent dans cette mesure un des éléments fondamentaux de la construction du socialisme. Cependant, si la «naturalisation» reçoit un soutien unanime, la manière dont elle serait censée se concrétiser suscite un sérieux contentieux. L'auteur montre bien que cette question influence la définition du socialisme et en particulier les formes de rétribution du travail ; selon la réponse apportée, le lien entre productivité et rémunération varie.

6 La deuxième partie du livre détaille la manière dont, au cours des années 1920, le communisme de guerre est devenu une construction historiographique. Le chapitre 5 analyse les discussions au sein du parti, ainsi que les principales études économiques et statistiques sur le communisme de guerre. Dans ce contexte, une attention particulière est accordée à G. Strumilin, économiste et statisticien, et notamment à son travail sur la dynamique des salaires et de la productivité en Russie entre 1913 et 1922. La chronologie permet d'anticiper la thèse principale de cet auteur selon lequel les pratiques du communisme de guerre s'inscriraient dans une dynamique de plus longue durée (guerre mondiale, guerre civile, inflation), et à l'échelle européenne. C'est donc dans la " grande transformation » de l'économie qu'il faut trouver la véritable explication du phénomène.

7 Inversement, les thèses « libérales » (Hayek, Mises, Brutskus), discutées dans le chapitre 6, estiment que le communisme de guerre et la planification expriment les distorsions de l'idéologie socialiste. De manière plus radicale encore, d'autres auteurs, influencés par Spengler (dont Salomoni retrace la réception en Russie), inscrivent ces expériences soviétiques dans le cadre de la décadence générale de l'Occident.

8 Le chapitre 7 détaille les approches « sociologiques » de cette thèse de la dégénérescence qui vont jusqu'à évoquer la notion de race (Sorokin). 
9 Enfin le chapitre 8 analyse la pensée d'un auteur aussi riche que négligé (à l'exception des travaux de Jutta Scherrer et, plus récemment, de Bordjugov et Biggart): Aleksandr Bogdanov. Selon cet auteur, le communisme de guerre exprime la " greffe » d'éléments socialistes sur le capitalisme qui donne ainsi vie à une «formation économiquemilitaire $»$.

10 L'ouvrage de Antonella Salomoni s'appuie sur une bibliographie très riche et sur une rigueur philologique extrême. Les notes permettent de reconstruire le " parcours » non seulement des auteurs, mais aussi de leurs œuvres (éditions, réception). C'est là, espérons-le, un premier pas, important, vers une approche de l'histoire soviétique qui, suivant l'enseignement de Marc Bloch, serait moins dictée par le besoin d'exprimer des jugements que par sa capacité à poser des questions. En particulier, à partir de cet ouvrage, au moins deux pistes de recherches à venir se dégagent. Il faudrait avant tout comprendre l'héritage de la pensée et celui de la Russie tsariste dans la mise en place de l'économie et de l'idéologie de la guerre civile. La réception de Marx en Russie (via la pensée allemande), les catégories et les études économiques du tournant du siècle présentent des spécificités qu'il serait important de mettre en rapport avec celles de la guerre civile.

11 En outre, il faudra se pencher sur les pratiques économiques concrètes du communisme de guerre telles qu'elles ressortent des archives, afin de comprendre les points de convergence, les décalages et les frictions avec la pensée et l'idéologie économiques si bien détaillées dans Il pane quotidiano. 